\title{
Implementación de planes de formación ciudadana: La experiencia regional de Curicó, 2017-2018
}

\author{
Citizen training plans: \\ The regional experience of Curicó, 2017-2018 \\ Jorge Molina Jara ${ }^{\mathrm{a}}$, María José Alcaíno JarA ${ }^{\mathrm{b}}$, \\ Miryam Aranda IturRiagac ${ }^{c}$ J JaCQUeline Morales CoRnejO $^{\mathrm{d}}$ \\ aUniversidad Católica del Maule, Curicó, Chile. \jmolina@ucm.cl \\ [orcid.org/0000-0002-8757-4909] \\ bUniversidad Católica del Maule, Curicó, Chile.ma.josealcaino@gmail.com \\ [orcid.org/0000-0002-8328-316X] \\ cUniversidad Católica del Maule, Curicó, Chile. miryam.aranda@gmail.com \\ [orcid.org/0000-0003-4031-5720] \\ dUniversidad Católica del Maule, Curicó, Chile. jacqueline.morales.co@gmail.com \\ [orcid.org/0000-0002-6775-8372]
}

\section{RESUMEN}

El trabajo analiza la elaboración e implementación de Planes de Formación Ciudadana de seis escuelas básicas urbanas de la ciudad de Curicó (Región del Maule, Chile), respondiendo a la Ley 20.911. Al respecto, esta investigación exploratoria entrevistó a directivos y analizó los planes elaborados por los establecimientos, constatando que muchos de ellos desarrollaban acciones de formación ciudadana previo a la Ley, que los planes fueron construidos sin participación de toda la comunidad, presentando distintos formatos y énfasis, y que las escuelas particulares subvencionadas desarrollaron mayor correspondencia con los objetivos de la Ley.

PALABRAS CLAVE: Formación ciudadana, Planes de Formación Ciudadana, Ley 20.911.

\section{ABSTRACT}

The research analyses the development and implementation of Citizen training Plans of six urban primary schools in the city of Curicó (Maule Region, Chile), responding to law 20,911. In this regard, this exploratory research interviewed headmasters and analyzed the plans prepared by the institutions, noting that many of them developed citizen formation actions prior to the law, that the plans were built without the participation of the entire community, presenting different formats and emphasis and that the subsidized schools developed greater correspondence with the objectives of the Law. 
KEY WORDS: Citizen Formation, Citizen training plans, Law 20.911.

\section{PROBLEMATIZACIÓN}

En el mes de abril del año 2016 fue publicada en el Diario Oficial la Ley 20.911, que estableció para todos los establecimientos educacionales reconocidos por el Estado, el deber de "incluir en los niveles de enseñanza parvularia, básica y media un Plan de Formación Ciudadana". Dicha Ley venía a complementar distintos marcos legales y curriculares, que reconocían en la educación un espacio clave en la generación de una ciudadanía democrática, participativa y respetuosa de los derechos humanos.

En la Ley se definió que el Ministerio de Educación pondría a disposición orientaciones para la creación e implementación de los Planes de Formación Ciudadana (en adelante PFC), lo que fue atendido por la entidad entre los meses de mayo del año 2016 y septiembre del año 2017, con tres documentos que orientan su elaboración (PNUD, 2018). No obstante, la misma Ley otorgaba libertad a las instituciones educativas para generar sus propios PFC, indicando: "cada sostenedor podrá fijar libremente el contenido del plan de formación ciudadana" (Ley 20.911, 2016), procurando considerar los objetivos de la Ley y de las bases curriculares vigentes. Esta indicación de la Ley abrió un amplio abanico de experiencias de PFC en el país, que creemos deben ser estudiadas.

Desde la entrada en vigencia de esta nueva Ley, han pasado 4 años -tiempo suficiente para iniciar una revisión de cómo las instituciones han atendido la obligación legal-. Por ello, pretendemos analizar cómo los establecimientos educacionales de la ciudad de Curicó han implementado los Planes de Formación Ciudadana estipulados en la Ley 20.911, buscando identificar cómo fueron construidos e implementados y verificar el nivel de cumplimiento de los objetivos de la Ley en las acciones estipuladas en los planes analizados.

Con respecto a la implementación de los PFC, recientemente han surgido algunas investigaciones en la ciudad de Santiago bajo la modalidad de tesis universitarias (Aguirre et al. 2016; Flores et al. 2017; Iturriaga, 2017; Ojeda, 2020; entre otros) y algunos artículos que dan cuenta de experiencias escolares, como el trabajo de Valentina Parra, centrado en un centro educativo de la comuna de Cerro Navia (2019) y el estudio que analiza la asesoría de académicos de la Universidad Católica Silva Henríquez, junto al Ministerio de Educación, a 48 escuelas pertenecientes a tres provincias de la Región Metropolitana para la implementación de sus PFC (Solorzano, 2019). Fuera de la Región Metropolitana los estudios son más bien escasos, destacando la publicación de Sixtina Pinochet y Javier Mercado (2018) centrada en escuelas de Antofagasta, la tesis de Maria José Alcaíno y colaboradores (2017) que recopila información de escuelas curicanas y la tesis universitaria de Daniela Hermosilla y Jennifer Sandoval (2019) enfocada en estudiar la implementación de los PFC en contextos escolares de la ciudad de Los Ángeles, Región del Bío-Bío.

Por su parte, en el año 2018, el Programa de las Naciones Unidas para el Desarrollo (en adelante PNUD) publicó el informe titulado: Estudio de Puesta en Marcha del Plan de Formación Ciudadana. En él busca dar cuenta de cómo ha comenzado la implementación de 
los PFC en el país, a partir de entrevistas y análisis de los planes de mejora, presentando una mirada global-nacional del proceso.

El estado de las investigaciones nos permite constatar que éstas aún son escasas, se concentran en la Región Metropolitana bajo la modalidad de tesis y que los estudios no dan cuenta de las experiencias de los establecimientos educacionales regionales, sobre la puesta en marcha de los PFC. Por ello, el presente trabajo viene a enfrentar esta merma y ampliar la mirada a través del análisis de la implementación de los PFC en colegios de Curicó, sorteando el centralismo o las miradas globales-nacionales de las investigaciones existentes.

La ciudad de Curicó se encuentra ubicada a 180 kilómetros al sur de Santiago, perteneciendo administrativamente a la Región del Maule, la cuarta región más poblada de Chile ( INE, 2017), tras las regiones Metropolitana, de Valparaíso y del Bío-Bío. La ciudad posee 149.136 habitantes ( INE, 2017), siendo la segunda más habitada de la región, tras la capital Talca, y "se encuentra dentro de las 20 ciudades más pobladas del país" (PLADECO, 2017, p. 31). En cuanto a las instituciones educativas de educación parvularia, básica y media, Curicó posee 99 establecimientos educacionales, la mayoría de ellos de dependencia Municipal (DAEM) y particular subvencionado, con 43 y 50 respectivamente, sólo 5 particulares pagados y 1 escuela de administración delegada.

\section{LA EMERGENCIA DE LA FORMACIÓN CIUDADANA}

En Chile, desde el año 1990 se viene consolidando una democracia representativa formal, respaldada en 16 eventos eleccionarios ${ }^{1}$ que han permitido escoger autoridades políticas y proyectar una estable transición democrática al mundo. Sin embargo, este aparente "exitoso" proceso transicional ha sido cuestionado por cientistas sociales, describiendo a la democracia chilena post dictadura como incompleta (Garretón\& Garretón 2010), semi soberana (Hunneus, 2014) o tutelada (Portales, 2000), por mantener enclaves autoritarios en la Constitución de 1980. Esta democracia incompleta propició una frágil democratización social que se ha expresado en una sostenida disminución de la participación ciudadana en espacios democráticos formales y una constante baja en la participación electoral (Paredes, 2011; PNUD, 2017), develando una inquietante desafección ciudadana con la democracia chilena y sus mecanismos eleccionarios.

En cifras, la situación es bastante expresiva de la tendencia a la baja en participación de la ciudadanía chilena. En efecto, en elecciones municipales "el porcentaje de votantes disminuyó del 79\% en 1992, al 45\% en 2012 y al 36\% en 2016. Lo mismo sucede en las elecciones de diputados, en las que se ha pasado de una participación en elecciones del 87\% en 1989, al 51\% en 2013" (PNUD, 2017, p. 13), dando cuenta de un sombrío panorama nacional.

Distintos autores (Dahl, 1986; Pateman, 2014; Corvalán \& Cox, 2015) sostienen que la participación es una condición necesaria del proceso de representación, y en Chile el número de votantes ha ido "de la mano con un descenso en otras expresiones de participación formal,

1 Entre 1989 y 2017 se desarrollaron 7 elecciones municipales (1992, 1996, 2000, 2004, 2008, 2012 y 2016), 7 elecciones presidenciales $(1989,1993,1999,2005,2009,2013$ y 2017) y 8 elecciones parlamentarias de Diputados y Senadores (1989, 1993, 1997, 2001, 2005, 2009, 2013 у 2017). 
como la militancia en partidos políticos, la filiación a sindicatos y otro tipo de asociaciones similares, y de un menor interés en la política en general” (Corvalán \& Cox, 2015, p. 177). La falta de participación en espacios formales ha ido provocando crisis de representación, la que a su vez confluye con diversos casos de corrupción, desigualdad y falta de transparencia (Bonometti \& Ruiz, 2010; Zovatto, 2014; Cox et al. 2014), los que, en conjunto, han ido erosionando la democracia representativa.

Esta desafección ciudadana con la democracia chilena -y con el sistema electoral- ha ido deslegitimando a las autoridades representativas y, con ello, el sistema político en su conjunto (PNUD, 2017). Ante este creciente divorcio entre la ciudadanía y la democracia, el Estado y sus instituciones fueron reaccionando con nuevos marcos legales y educativoscurriculares que buscan propiciar la valoración de la democracia y la formación de ciudadanos con sello participativo, aunque esquivando un cambio constitucional.

En efecto, el sistema político visualizó medidas remediales a la crisis de la desafección con la democracia, evitando cuestionamientos de fondo en torno a la legitimidad de la constitución, o a los límites que establece a la soberanía (Hunneus, 2014), propiciando, en cambio, modificaciones dentro del marco constitucional vigente, expresado en leyes y reformas curriculares educativas para enfrentar la problemática.

En términos legales, será sólo el año 2005 en que se suprimen los Senadores Designados y Vitalicios (Ley 20.050) quienes, al ser investidos por instituciones del Estado y no electos por sufragio popular, alteraban la voluntad ciudadana en el Congreso. Por su parte, el año 2011 se promulga la iniciativa legal (Ley 20.500) orientada a estimular la participación ciudadana y normar las organizaciones de la sociedad civil; al año siguiente, en enero de 2012, fue promulgada la Ley 20.568 que facilitó la inscripción automática en los registros electorales (a quienes cumplan los requisitos) y el voto voluntario. Posteriormente, el año 2015 se modifica el cuestionado sistema binominal para escoger a los representantes parlamentarios y municipales (Ley 20.840), y recientemente se realiza una reforma constitucional que limita la reelección indefinida de parlamentarios y representantes municipales (Ley 21.238), buscando garantizar la alternancia en el poder.

En el ámbito educativo, la Ley Orgánica Constitucional de Enseñanza (LOCE) promulgada el último día de la dictadura cívico-militar se mantuvo hasta el año 2009, cuando fue reemplazada por la Ley General de Educación (LGE). Por sus definiciones, la LOCE formalizó un "mercado educativo sometido a reglas generales de regulación" (UNESCO, 2018, p. 19), consolidando en la década del noventa un ciudadano "despolitizado e individualizado que se había instalado como objetivo final” (Mayorga, 2019, p. 362). Por ello, no extrañaron los magros resultados de estudiantes chilenos en pruebas y ranking internacionales que analizan los conocimientos en educación cívica, lo que para el investigador Isidoro González Gallegos hizo reaccionar al Estado chileno, "dando un paso hacia arriba y creando para el año 2004 la Comisión Nacional de Formación Ciudadana” (González, 2019, p. 9) e impulsó varios cambios curriculares, para la formación de una ciudadanía democrática.

La creación de esta comisión y las definiciones que adoptó posibilitaron ir supliendo graves omisiones heredadas de la dictadura cívico-militar chilena, cuyo currículo fue deficitario respecto de la consideración de algunos ejes claves asociados a la democracia y a 
los derechos humanos, así como a otros relacionados con el aprendizaje de vida en común (Muñoz et al. 2010). No obstante, será entre el año 2009 y 2012 cuando se consolida el enfoque de Formación Ciudadana a través del establecimiento de nuevas bases curriculares, incorporándolo en los Objetivos de Aprendizajes Transversales (OAT) para las distintas áreas del conocimiento y estableciéndolo como eje en las asignaturas de Orientación e Historia, Geografía y Cs. Sociales.

Estos cambios educativos y legales desarrollados en las últimas décadas se buscaron robustecer debido a que las evaluaciones internacionales mostraban a Chile aún con déficit en esta materia. Para ello, el gobierno de Michelle Bachelet envía un proyecto de Ley, indicando: "a pesar de la presencia de la Formación Ciudadana en el currículum del sistema escolar, la necesidad de fortalecer ésta es evidente si se considera la información disponible sobre los aprendizajes alcanzados por los estudiantes chilenos en esta área" (Mensaje en Sesión 23. Legislatura 363. 8 de mayo de 2015).

Así, tras el debate propio de los parlamentarios, fue promulgada el año 2016 la Ley 20.911, que declara como propósito "contribuir hacia la formación de ciudadanos, con valores y conocimientos que fomenten el desarrollo del país, con una visión del mundo centrada en el ser humano, como parte de un entorno natural y social". De esa manera, se instituye la obligatoriedad de establecer un Plan de Formación Ciudadana en todos los establecimientos educacionales reconocidos por el Estado, mientras que en $3^{\circ}$ y $4^{\circ}$ medio, a contar del 2017 se implementará la asignatura de Formación Ciudadana.

\section{METODOLOGÍA DE ESTUDIO}

Al ser la Ley 20.911 una obligación reciente para los establecimientos educacionales, Curicó y la Región del Maule carecían de investigaciones que dieran cuenta del proceso, por ello el presente trabajo es cualitativo de carácter exploratorio, ya que se introdujo en analizar la implementación de los PFC en escuelas de la ciudad.

$\mathrm{Al}$ momento de catastrar las instituciones que serían incluidas en la investigación, se pudo establecer que la ciudad de Curicó contaba con 99 establecimientos educacionales (PLADECO 2017), tanto de educación parvularia, educación básica y media, pero al focalizar la muestra únicamente en escuelas básicas del radio urbano y que tuviesen PFC, ésta se redujo a 31 de ellos. Sin embargo, tras ser invitados a participar de esta investigación, únicamente 6 establecimientos (3 municipales y 3 subvencionados) accedieron a ser incluidos, facilitando sus Planes de Formación Ciudadana, estando dispuestos sus directivos a participar de una entrevista semiestructurada y firmando un consentimiento informado, lo que condiciona la muestra final de esta investigación.

Para enfrentar la entrevista y los PFC, se construyeron dos instrumentos: un guión de preguntas para la entrevista semiestructurada que fue validada por expertos en el área educativa ${ }^{2}$ y una matriz de análisis para los PFC, donde se asignó a cada uno de los 9 objetivos

Leyla Danae Torres Bravo, Profesora de Historia y Geografía, y Doctora en Teoría y Métodos educativos; Macarenna Javiera Astorga Poblete, Psicóloga y Magíster en Salud Mental Infanto Juvenil; Enrique Muñoz Reyez, Profesor de Historia y Geografía, y Doctor en Didáctica de la Historia, la Geografía y las Ciencias Sociales. 
de la Ley 20.911 una categoría, para verificar que las acciones contenidas dentro de los 6 planes estudiados cumplieran de manera efectiva con la Ley y sus objetivos.

El instrumento de entrevista semiestructurada fue integrado a una matriz de análisis que recoge las impresiones de los directivos y/o encargados del Plan. Las preguntas del presente instrumento fueron encasilladas en dos categorías: Elaboración del Plan y Opinión Personal. Además, cada una de las seis preguntas fue clasificada en una subcategoría distinta, siendo reducida a uno o dos conceptos (Tabla 1).

Tabla 1. Categorización de las preguntas de la entrevista.

\begin{tabular}{clc}
\hline Categoría & \multicolumn{1}{c}{ Pregunta } & Subcategoría \\
\hline $\begin{array}{c}\text { Elaboración } \\
\text { del Plan }\end{array}$ & $\begin{array}{l}1-\text { ¿Desde qué fecha el establecimiento cuenta con su Plan de Formación } \\
\text { Ciudadana? }\end{array}$ & Iniciación \\
\hline & $\begin{array}{l}\text { 2- ¿Cuál fue el procedimiento utilizado para elaborar el Plan de Formación } \\
\text { Ciudadana? ¿Quiénes participaron? }\end{array}$ & Elaboración \\
\hline & $\begin{array}{l}\text { 3- ¿Cuál es el objetivo del Plan formulado por el establecimiento? ¿Cómo se } \\
\text { vincula con su proyecto educativo? }\end{array}$ & Intencionalidad \\
\hline & $\begin{array}{l}\text { 5- ¿Cómose está ejecutando elPlandeFormaciónCiudadana enelestablecimiento? } \\
\text { (Especificar el tiempo destinado, recursos materiales y humanos) }\end{array}$ & Ejecución \\
\hline Personal & $\begin{array}{l}\text { 4- ¿De qué manera considera usted que el Plan de Formación Ciudadana } \\
\text { contribuye al fortalecimiento de la democracia? }\end{array}$ & Contribución \\
\hline & $\begin{array}{l}\text { 6- ¿Cuál es su apreciación sobre la obligatoriedad de la implementación del } \\
\text { Plan de Formación Ciudadana en los establecimientos del país? }\end{array}$ & Obligatoriedad
\end{tabular}

Fuente: Elaboración propia.

En segundo lugar, los PFC proporcionados por los seis establecimientos educacionales que participan de la muestra (De dependencia municipal: Escuela Cataluña, Colegio El Boldo, Colegio Polivalente Japón; de carácter particular subvencionado: Centro Educacional Monseñor Manuel Larraín Errázuriz, Colegio Alta Cumbre y Colegio San Marcos), fueron sometidos a una matriz de análisis, en la que cada objetivo de la Ley 20.911 tendrá una categoría distinta, siendo reducido a uno o más conceptos (Tabla 2).

\section{ANÁLISIS DE LOS HALLAZGOS}

\section{Las Entrevistas}

Los seis establecimientos participantes de la investigación proporcionaron información que fue analizada en la matriz $\mathrm{n}^{\circ} 1$, entregando los siguientes resultados por categorías: Elaboración del Plan y Opinión Personal; y las subcategorías: iniciación, elaboración, intencionalidad, ejecución, contribución y obligatoriedad.

En la categoría de la Elaboración del Plan, se logró recabar antecedentes relevantes del proceso de levantamiento del PFC de cada establecimiento. En la subcategoría iniciación, centrada en conocer desde cuándo comenzaron el PFC, cinco de los seis establecimientos afirmaron que 
Tabla 2.Categorización de los objetivos del Plan.

a) Promover la comprensión y análisis del concepto de ciudadanía y los derechos y deberes asociados a ella, entendidos éstos en el marco de una república democrática, con el propósito de formar una ciudadanía activa en el ejercicio y

Ciudadanía activa cumplimiento de estos derechos y deberes.
b) Fomentar en los estudiantes el ejercicio de una ciudadanía crítica, responsable, respetuosa, abierta y creativa.
Ciudadanía consciente

c) Promover el conocimiento, comprensión y análisis del Estado de Derecho y de la Institucionalidad local, regional y nacional, y la formación de virtudes cívicas en los estudiantes.

Estado de Derecho

d) Promover el conocimiento, comprensión y compromiso de los estudiantes con los derechos humanos reconocidos en la Constitución Política de la República y en los tratados internacionales suscritos y ratificados por Chile, con especial énfasis

Derechos Humanos en los derechos del niño.

e) Fomentar en los estudiantes la valoración de la diversidad social y cultural del país.

Diversidad

\begin{tabular}{llc}
\hline f) & Fomentar la participación de los estudiantes en temas de interés público. & Participación civil \\
\hline g) & Garantizar el desarrollo de una cultura democrática y ética en la escuela. & Cultura democrática \\
\hline h) & Fomentar una cultura de la transparencia y la probidad. & $\begin{array}{c}\text { Transparencia y } \\
\text { probidad }\end{array}$ \\
\hline i) & Fomentar en los estudiantes la tolerancia y el pluralismo. & Tolerancia y pluralismo
\end{tabular}

Fuente: Elaboración propia.

durante el año 2016 empezaron a instaurar acciones para la elaboración de sus planes, sin embargo, mencionan que desplegaron iniciativas tendientes a la formación ciudadana antes de que la Ley les obligase. Al respecto, la directora del Colegio San Marcos, Olivia González, señaló:

"Las actividades relacionadas con la formación ciudadana se han hecho desde hace mucho tiempo atrás. Desde antes se hacían cosas, a lo mejor no como Ley, pero sí se trabaja constantemente en el colegio en general lo que es la formación ciudadana. Porque esto es un tema transversal, así que siempre se está trabajando, a lo mejor ahora sí se hace más sistemático...”

Por otro lado, la subcategoría que apunta a la elaboración del Plan de Formación Ciudadana, cuatro de los seis establecimientos delegaron la misión de la creación del PFC a los docentes (destacando los de Historia, Geografía y Ciencias Sociales), en estrecha colaboración con los directivos. El director del Colegio El Boldo describió el proceso mencionando: “(...) la profesora de Historia, ella me entregó objetivos y yo hice lo medular del plan, fuimos dos los que participamos, pero después se fue incorporando a fines del 2016 la otra profesora de Historia" (Luis Mosquera, Director Colegio El Boldo). 
Por su parte, desde el Colegio San Marcos indicaron: "partimos por una conversación con la Ley, con las profesoras de Historia. Después, con las profesoras de Historia se elaboró una síntesis, así como resumen del proyecto y eso se traspasó al consejo de profesores" (Olivia González, Directora Colegio San Marcos).

Las orientaciones emanadas desde el Ministerio de Educación esperaban que la creación de los PFC "idealmente sea el resultado de un proceso de reflexión comunitaria (directivos, estudiantes, familias, docentes y asistentes de la educación)" (MINEDUC, 2016, pp. 21-22); sin embargo, los hallazgos en las escuelas de Curicó evidencian que este anhelo ministerial no se cumplió, encabezando dicha tarea profesores y directivos de los establecimientos, con algunas interacciones con grupos focales (consejos de profesores o estudiantes), pero no se logró constatar una reflexión y participación de la comunidad en su conjunto. La excepción a esta tendencia la representó el Colegio Alta Cumbre, al contemplar a la comunidad educativa en la elaboración del plan:

“(...) trabajamos con distintos grupos, con el centro general de padres, con todos los padres que quisieran participar, con los alumnos de media que quisieran participar e hicimos focus group también con los profesores, con los funcionarios en general, viendo cómo podíamos potenciar nuestro proyecto educativo" (Carmen Ormazábal, Rectora Colegio Alta Cumbre).

En el caso de la subcategoría de intencionalidad, que apunta a determinar el objetivo del plan y su vinculación con los proyectos educativos, es posible destacar que todos los participantes de la muestra utilizaron el PFC para unificar los aspectos valóricos considerados en su PEI, destacando el respeto como un factor preponderante en la formación de sus estudiantes. En la Escuela Cataluña se indicó: "el objetivo del plan es desarrollar las competencias sociales de los estudiantes, desarrollar la participación, los deberes y los derechos, a resolver los conflictos" (Marcia González, UTP Escuela Cataluña). Por su parte, desde el Colegio Polivalente Japón afirmaron: "tenemos que basar en los objetivos que están en la Ley y de acuerdo a eso establecer acciones, entonces eso fue lo que hicimos estableciendo acciones y todas van relacionadas con nuestro proyecto educativo" (Jorge Rojas, Director Colegio Japón).

Importante en esta pesquisa fue constatar las diferencias que presentaron los establecimientos al ejecutar sus planes. Esto se puede entender desde la misma normativa legal que asigna responsabilidad a cada sostenedor para que elabore sus propios planes. Por ello, la información recabada en la subcategoría de ejecución refleja la diversidad que la Ley permitió.

En general, las actividades que potenciaron la implementación del plan, tales como: diarios murales, debates, elección de Directivas de Alumnos y de Centro General de Padres, fueron consideradas instancias para promover la participación ciudadana de las comunidades educativas. El Colegio Alta Cumbre innovó al incorporar una nueva asignatura llamada Formación Ciudadana y Valórica. Esta fue incluida en su plan de estudio con dos horas pedagógicas, buscando potenciar aspectos democráticos y valóricos en la formación de sus estudiantes. 
"Queremos alumnos reflexivos, queremos alumnos solidarios, entonces todo lo que se hace extraescolar se hace con una mirada de formación integral del alumno, y luego la asignatura de Formación Ciudadana y Valórica, que está basada en todo lo que nuestro PEI definió que quería formar en los alumnos" (Carmen Ormazábal, Rectora Colegio Alta Cumbre).

En cuanto a la contribución del PFC a sus PEI, todos los entrevistados hacen referencia al positivo aporte que realiza en la difusión de sus valores institucionales, como así también en el desarrollo de habilidades comunicativas, que permiten a los estudiantes expresar sus opiniones sobre temas contingentes, cultivando el respeto y la tolerancia.

"Muchas de las actividades implican que el alumno participe de sus opiniones de manera fundamentada (...) uno de los sellos nuestros, que los alumnos cuando salgan de aquí, sean capaces de pararse, de ser buenos expositores, de poder plantearse frente a un público, que no tengan ese temor" (Lida Rubilar, UTP Centro Educacional Manuel Larraín).

Finalmente, en relación a la obligatoriedad de los PFC, los entrevistados entregaron sus apreciaciones favorables a la Ley y sus objetivos, indicando:

“...a mí me parece muy bien, muy bien, me parece que es un aspecto básico en la formación de personas (...) la formación valórica y ciudadana, nosotros le agregamos "valórica", porque claro yo puedo tratar de formar ciudadanos, pero yo los quiero empoderados, con valores sólidos” (Carmen Ormazábal, Rectora Colegio Alta Cumbre).

La obligatoriedad que establece la Ley implicó que todos los establecimientos educacionales chilenos cumplieran con acciones que propicien la cultura participativa de los estudiantes. Jorge Rojas, director del Colegio Japón, realizó una autocrítica sobre lo desprovista que estuvieron las escuelas chilenas de valores democrático-ciudadanos y cómo la Ley 20.911 llegó a suplir esta carencia: "si tú no lo haces Ley, si tú no lo haces obligatorio, muchos colegios no tendrían plan y no trabajarían este tema".

\section{Análisis de los Planes de Formación Ciudadana}

El análisis de los PFC de los colegios que componen la muestra se desarrolló tras categorizar en conceptos los objetivos de la Ley, permitiendo verificar si las iniciativas desarrolladas eran coherentes con el mandato legal.

El primer objetivo apunta al logro de la ciudadanía activa. La mayoría de los establecimientos presenta iniciativas enfocadas en elecciones democráticas (centro de alumnos y directivas de curso), también en difundir información de las elecciones presidenciales, desarrollar unidades o talleres relacionados al análisis de conceptos cívicos y debates. Dentro de las estrategias planteadas, 4 de 6 escuelas cumplen con el objetivo. En 
esta línea, el Colegio Polivalente Japón presentó una lúdica actividad titulada: "Construcción de juegos con conceptos políticos, autoridades e instituciones del Estado, para estudiantes del segundo ciclo básico".

El segundo objetivo de la Ley se enfoca en la ciudadanía consciente, procurando potenciar el correcto ejercicio de la ciudadanía. Los PFC analizados reflejaron diversas iniciativas: elecciones internas, cuidado del medio ambiente, salidas pedagógicas y debates. A pesar de lo diverso de sus iniciativas, 4 de los 6 colegios logran el objetivo.

El tercer objetivo apunta hacia el conocimiento sobre el Estado de derecho, y es abordado con acciones que nuevamente se materializan en elecciones internas, murales cívicos e informativos y organigrama de las instituciones. Sólo 3 de 6 colegios cumplen con iniciativas para el objetivo mencionado.

El cuarto objetivo de la Ley busca promover el conocimiento, comprensión y compromiso de los estudiantes con los derechos humanos. En este objetivo, los PFC transparentaron una preocupante situación asociada a la reducción de los derechos humanos, abordando únicamente los derechos del niño. La excepción a esta constatación la entregó el Colegio San Marcos, quienes desarrollan "la semana de ciudadanía y los DD.HH.", donde presentan una feria en torno a esta temática y debates focalizados en los 17 objetivos de la ONU.

El quinto objetivo de la Ley, centrado en fomentar la valoración de la diversidad, es el que mayor presencia tiene en los PFC analizados, pues la totalidad de los colegios desarrolla estrategias enfocadas en su cumplimiento. Si bien existe una multiplicidad de iniciativas, desde muestras folclóricas, semana de las artes y la cultura, semana del patrimonio cultural, feria intercultural, día nacional de los pueblos originarios, mes de la región del Maule, galas artísticas recorriendo Chile y salidas pedagógicas, todas ellas establecen la valoración de la diversidad como eje central.

En el sexto objetivo de la Ley, asociado a la participación civil, los PFC reflejan interés en fomentar la preocupación por lo público, implementando ficheros informativos, debates, programas radiales, efemérides y vinculación con el medio ambiente. En el logro de este objetivo, el Colegio Polivalente Japón establece una vinculación con la comunidad a través de una jornada de limpieza de calles, veredas y recolección de basura de las poblaciones que rodean la escuela.

En el desarrollo de una cultura democrática y ética en la escuela, 4 de los 6 establecimientos cumplieron con el logro de este objetivo, a través de la promoción de talleres artístico-deportivos, elecciones de centro de estudiantes, elecciones de directivos de curso y jornadas de reflexiones.

El octavo objetivo busca fomentar una cultura de transparencia y probidad. En esta subcategoría la mayoría de la muestra analizada no cumple con este propósito, pues sólo 2 de los 6 colegios sí lo contemplan, dando cuenta de una inquietante despreocupación por estos temas. Las escuelas que sí lo abordan, desarrollan cuenta pública de su gestión ante los centros de padres y estudiantes, fomentando la transparencia en la comunidad educativa. De igual manera, uno de los establecimientos consigna una charla de educación financiera dirigida a los apoderados, para propiciar el adecuado uso de los recursos y la honradez en su gestión. 
El noveno objetivo está centrado en la tolerancia y el pluralismo, buscando que los establecimientos instalen experiencias que permitan a su comunidad vivenciar la coexistencia con otras culturas y visiones políticas y evitar prácticas excluyentes e intolerantes. Al respecto, la muestra permitió constatar el desarrollo de actividades folclóricas, día nacional de los pueblos originarios, feria intercultural y encuentros con otras realidades sociales a través de visitas solidarias. Sin embargo, en el ámbito del pluralismo no se pudo constatar estrategias asociadas al objetivo.

Es importante mencionar que no todos los establecimientos cumplen con la totalidad de los objetivos, así como también no todos presentaron el mismo formato de Plan de Formación Ciudadana. Esto, debido a que el Ministerio de Educación entregó libertad para la creación del Plan y las orientaciones funcionaron como apoyo y no como una estructura obligatoria.

Los hallazgos realizados tras los análisis de los Planes de Formación Ciudadana de los colegios participantes nos permitieron, además, constatar que las escuelas subvencionadas tuvieron mayor correspondencia con los objetivos de la Ley por sobre las escuelas municipales (Tabla 3$)^{3}$.

Tabla 3. Cuadro resumen de niveles de logro.

\begin{tabular}{|c|c|c|c|c|c|c|}
\hline $\begin{array}{l}\text { Colegio/ } \\
\text { Objetivo }\end{array}$ & $\begin{array}{l}\text { Colegio } \\
\text { El Boldo }\end{array}$ & $\begin{array}{l}\text { Escuela } \\
\text { Cataluña }\end{array}$ & $\begin{array}{l}\text { Colegio } \\
\text { Polivalente } \\
\text { Japón }\end{array}$ & C.E.M.M.L.E & $\begin{array}{c}\text { Colegio } \\
\text { San Marcos }\end{array}$ & $\begin{array}{l}\text { Colegio } \\
\text { Alta } \\
\text { Cumbre }\end{array}$ \\
\hline Objetivo 1: Ciudadanía activa & $\mathrm{L}$ & ML & $\mathrm{L}$ & ML & $\mathrm{L}$ & $\mathrm{L}$ \\
\hline $\begin{array}{l}\text { Objetivo 2: Ciudadanía } \\
\text { consciente }\end{array}$ & $\mathrm{L}$ & ML & ML & $\mathrm{L}$ & $\mathrm{L}$ & $\mathrm{L}$ \\
\hline Objetivo 3: Estado de derecho & $\mathrm{L}$ & NL & $\mathrm{L}$ & $\mathrm{L}$ & ML & ML \\
\hline Objetivo 4: Derechos Humanos & ML & NL & NL & ML & $\mathrm{L}$ & NL \\
\hline Objetivo 5: Diversidad & $\mathrm{L}$ & $\mathrm{L}$ & $\mathrm{L}$ & $\mathrm{L}$ & $\mathrm{L}$ & $\mathrm{L}$ \\
\hline Objetivo 6: Participación civil & ML & NL & ML & $\mathrm{L}$ & $\mathrm{L}$ & NL \\
\hline $\begin{array}{l}\text { Objetivo 7: Cultura } \\
\text { democrática }\end{array}$ & NL & $\mathrm{L}$ & $\mathrm{L}$ & $\mathrm{L}$ & $\mathrm{L}$ & NL \\
\hline $\begin{array}{l}\text { Objetivo 8: Transparencia y } \\
\text { probidad }\end{array}$ & $\mathrm{L}$ & NL & NL & $\mathrm{L}$ & NL & NL \\
\hline $\begin{array}{c}\text { Objetivo 9: Tolerancia y } \\
\text { pluralismo }\end{array}$ & ML & ML & NL & $\mathrm{L}$ & $\mathrm{L}$ & $\mathrm{L}$ \\
\hline
\end{tabular}

Fuente: Elaboración propia.

3 Cabe destacar que se categorizaron las iniciativas en: Logrado, Medianamente logrado y No logrado.

Logrado: iniciativas que cumplen a cabalidad con el objetivo de la Ley 20.911.

Medianamente logrado: cuando las estrategias cumplen parcialmente con el objetivo de la Ley 20.911.

No logrado: cuando el establecimiento no presenta estrategia, o bien no cumple con el objetivo de la Ley 20.911. 


\section{CONCLUSIONES}

Los déficits en conocimiento cívico, valoración de la democracia y participación en comunidad de jóvenes chilenos, constatados en pruebas internacionales y la sostenida disminución de la participación en procesos eleccionarios, daban cuenta del desconocimiento y desafección con la democracia y sus mecanismos de elección de representantes. Ante ello, el Estado y el sistema político reaccionaron con marcos legales y educativo-curriculares que pusieron acento en propiciar la democracia y la generación de una ciudadanía activa y respetuosa de los derechos humanos, encontrando en los establecimientos educacionales y el enfoque de formación ciudadana el espacio propicio para ello.

La Ley 20.911 complementa los esfuerzos educativos curriculares, posibilitando el involucramiento de la comunidad educativa en el interés por lo público y la democracia. Con respecto a este nuevo marco legal, el estudio de lo acaecido en la ciudad de Curicó representa una mirada regional que releva la experiencia de establecimientos educacionales que asimilan, construyen e implementan Planes de Formación Ciudadana.

Mediante entrevistas y análisis de los planes de 6 establecimientos urbanos de educación básica, se constató que para las comunidades educativas la promulgación de esta ley resultó beneficiosa para la formación integral de los estudiantes, la inserción de valores democráticos y que era una falencia del sistema educativo chileno, urgente de superar.

Además que, en el período de análisis de los colegios auditados (2017-2018), los particulares subvencionados fueron aquellos que en mayor medida cumplieron con los objetivos de la Ley. También fue posible constatar que los establecimientos de este estudio desarrollaban experiencias de formación democrática a través de sus Proyectos Educativos Institucionales (PEI) en coordinación con su Proyecto de Mejoramiento Educativo (PME), por lo que este marco legal viene a complementar el deseo de fortalecer las actitudes cívicas y democráticas de los estudiantes.

Es necesario destacar que los colegios que participaron de la investigación tuvieron una positiva acogida a esta ley, sin embargo, el diseño y creación de los planes no incluyó a la comunidad educativa en su totalidad, siendo asumida esta labor principalmente por docentes, junto a directivos y algunos grupos focalizados. Esta realidad coincide con las verificadas por el "Estudio de Puesta en Marcha del Plan de Formación Ciudadana" elaborado por el PNUD, quienes además pesquisaron una gran diversidad de formatos y énfasis en los PFC, situación constatada por el estudio en Curicó.

Tanto las escuelas analizadas como el mencionado informe del PNUD, apuntan a que el seguimiento que se haga del PFC es clave para evaluar si contribuye al logro de los objetivos planteados en la Ley, así como para reformular acciones a futuro.

Finalmente, y como reflexión complementaria, lo acaecido tras el "estallido social" del 18 de octubre y las consecuencias sanitarias de la pandemia, dejan una serie de interrogantes sobre las nuevas formas de participación ciudadana desplegadas en este contexto, y cómo las comunidades educativas junto a los PFC dialogan con estas nuevas experiencias organizativas que, al incorporar redes sociales, posibilitan deliberar, criticar, proponer y comprometerse con sus comunidades. Aquí las escuelas, la formación ciudadana y los PFC tienen un desafío 
IMPLEMENTACIÓN DE PLANES DE FORMACIÓN CIUDADANA

en asimilar estas novedosas formas de participación que hoy se masifican, y que incluyen espacios de ciudadanía digital.

\section{REFERENCIAS}

Aguirre, D., Domínguez, M., Olivares, F., Orellana, M., Pedraza, F., Peñaloza, S., Valdés, P., \& Valenzuela, C. (2016). Formación ciudadana en el sistema escolar chileno. Aplicación de la Ley 20.911 al Colegio Metodista de Santiago. Seminario de grado para optar al grado de Licenciatura en Educación y Título profesional de Pedagogía en Historia y Geografía. Universidad Católica Silva Henríquez. Santiago.

Alcaíno M., Aranda, M., \& Morales, J. (2017). Análisis sobre la implementación del Plan de Formación Ciudadana en los establecimientos educacionales de Curicó. Seminario para optar al Título de Profesor de Educación General Básica con Mención en Historia, Geografía y Ciencias Sociales. Universidad Católica del Maule. Curicó.

Bachelet, M. (2015) Mensaje de S.E. la Presidenta de la República con el que inicia un proyecto de ley que crea el Plan de Formación Ciudadana para los establecimientos educacionales reconocidos por el Estado. Santiago. Recuperado de https://www.bcn.cl/ historiadelaley/historia-de-la-ley/vista-expandida/4891/

Bonometti, P, \& Ruiz, S. (2010). La democracia en América Latina y la constante amenaza de la desigualdad. Andamios, 7, 11-36. Recuperado de: http://www.scielo.org.mx/scielo. php?script=sci_arttext\&pid=S1870-00632010000200002\&lng=es\&tlng=es.

Centro Educacional Monseñor Manuel Larraín Errazuriz (2017). Plan de Formación Ciudadana. Curicó.

Colegio Alta Cumbre (2016). Plan de Formación Ciudadana. Curicó.

Colegio El Boldo (2017). Plan de Formación Ciudadana. Curicó.

Colegio Polivalente Japón (2017). Plan de Formación Ciudadana. Curicó.

Colegio San Marcos (2016). Plan de Formación Ciudadana. Curicó.

Corvalán, A., \& Cox, P. (2015). Participación y desigualdad electoral en Chile. En C. Cox, \& J. C. Castillo (Eds.), Aprendizaje de la ciudadanía: contextos, experiencias y resultados (pp. 175-206). Centro de Estudio de Políticas y Prácticas en Educación-Ediciones UC. Santiago.

Cox, C., Bascopé, M., Castillo, J. C., Miranda, D., \& Bonhomme, M. (2014). Educación ciudadana en América Latina: Prioridades de los currículos escolares. IBE Working Papers on Curriculum Issues, 14, 1-42.

Dahl, R. (1986). Democracy, liberty, and equality. Oxford University Press, New York. Escuela Cataluña (2017). Plan de Formación Ciudadana. Curicó.

Flores, N., Guzmán, M., Márquez, A., \& Rojas, M. (2017). Formación Ciudadana Propuesta para la evaluación de la implementación de los Planes de Formación Ciudadana del Departamento Provincial oriente de Santiago. Seminario de grado para optar al grado de Licenciatura en Educación y Título profesional de Pedagogía en Historia y Geografía. Universidad Católica Silva Henríquez. Santiago.

Garretón, M., \& Garretón, R. (2010). La democracia incompleta en Chile: La realidad tras 
los rankings internacionales. Revista de Ciencia Política. 30(1). 115-148. https://doi. org/10.4067/S0718-090X2010000100007

González, I. (2019). Prólogo a la formación ciudadana: sus temas, sus escenarios y las propuestas para el desarrollo vistos desde el viejo continente. En C. Muñoz \& B. Torres (Eds.), Escuela y Formación Ciudadana: temas, escenarios y propuestas para su desarrollo (pp. 7-15). Universidad de Concepción. Concepción.

González, M. (7 de octubre de 2017). Entrevistada por M. Aranda. Audio digital. Curicó. Gonzalez, O. (10 de octubre de 2017). Entrevistada por M. Aranda. Audio digital. Curicó. Hunneus, C. (2014). La democracia semisoberana. Editorial Taurus. Santiago.

Hermosilla D., \& Sandoval, J. (2019). El Plan de Formación Ciudadana a dos años de su implementación. Seminario de Título para optar al grado de Licenciado en Educación y al Título Profesional de Profesor de Educación General Básica Especialidad en Lenguaje y Ciencias Sociales. Universidad de Concepción. Los Ángeles.

ILUSTRE MUNICIPALIDAD DE CURICÓ (2017). Plan de desarrollo comunal. Curicó.

Iturriaga, J. (2017). Percepción de las y los estudiantes de tercer año medio sobre ciudadanía y su relación con el plan de formación ciudadana del Liceo Indira Gandhi implementado en año 2017. Comuna La Florida. Tesis para optar al grado académico de Magíster en Ciencias de la Educación con Mención en Currículum y Evaluación, Escuela de Educación, Universidad Mayor. Santiago.

INSTITUTO NACIONAL DE ESTADÍSTICA (2017). Síntesis de resultados CENSO 2017. Santiago. Ley N²0.050. Diario Oficial de la República de Chile, Santiago, 26 de agosto de 2005.

Ley N²0.500. Diario Oficial de la República de Chile, Santiago, 16 de febrero de 2011. Ley N²0.568. Diario Oficial de la República de Chile, Santiago, 31 de enero de 2012. Ley N²0.840. Diario Oficial de la República de Chile, Santiago, 05 de mayo de 2015. Ley N²0.911. Diario Oficial de la República de Chile, Santiago, 02 de abril de 2016. Ley N²1.238. Diario Oficial de la República de Chile, Santiago, 08 de julio de 2020. Mayorga, R. (2019). Una red educativa, cuatro escuelas, millones de ciudadanos: Educación, Estado republicano y sociedad civil en Chile (1813-2011). En I. Jaksic \& F. Rengifo (Eds.), Historia Política de Chile, 1810-2010. Tomo II Estado y Sociedad (pp . 333-365). Fondo de Cultura Económica y Universidad Adolfo Ibáñez. Santiago.

MINISTERIO DE EDUCACIÓN (2016). Orientaciones para la elaboración del Plan de Formación Ciudadana. Santiago.

Mosquera, L. (9 de octubre de 2017) Entrevistado por M. Alcaíno. Audio digital. Curicó.

Muñoz, C., Vásquez, N., \& Reyes, L. (2010). Percepción del estudiantado de enseñanza básica sobre el rol del Estado, las instituciones públicas, la democracia, la ciudadanía y los derechos de las mujeres y de los inmigrantes. Estudios Pedagógicos, 36. 153-175. Recuperado de http://mingaonline.uach.cl/pdf/estped/v36n2/art09.pdf.

Ojeda, P. (2020). Diseño de un diagnóstico participativo para la elaboración del plan de formación ciudadana. Experiencia en un colegio de la comuna de El bosque. Proyecto de Magíster presentado a la Facultad de Educación de la Pontificia Universidad Católica de Chile para optar al grado de académico de Magíster en Educación mención Evaluación. Universidad Católica de Chile. Santiago. 
Ormazabal, C. (6 de octubre de 2017). Entrevistada por M. Alcaino. Audio digital. Curicó.

Paredes, J. (2011). Ciudadanía, Participación y Democracia Deuda y Déficit en los 20 años de "Democracia" en Chile. Polis. 10(28). 473-499. https://doi.org/10.4067/S071865682011000100022

Parra, V. (2019). La investigación histórica en la escuela: experiencia y resultados de su implementación como estrategia y acción pedagógica del Plan de Formación Ciudadana. Cuadernos de Historia de la Educación, 12. 68-90.

Pateman, C. (2014). Participación y teoría democrática. Editorial Prometeo. Buenos Aires.

Pinochet, S., \& Mercado J. (2018). Construyendo Ciudadanía: La experiencia de Antofagasta frente a los Planes de Formación Ciudadana. En B. Tosar, A. Santisteban \& J. Pagès (Eds.), ¿Què està passant al món? ¿Què estem ensenyant? Per un ensenyament de les ciències socials centrat en els problemes, la justícia social i la ciutadania global (pp. 147155). GREDICS- UAB. Barcelona.

PNUD (2017). Diagnóstico sobre la participación electoral en Chile. Proyecto Fomentando la participación electoral en Chile: por una ciudadanía más involucrada y participativa. Santiago.

PNUD (2018). Estudio de Puesta en Marcha del Plan de Formación Ciudadana. Informe elaborado por el Proyecto Educación Programa de las Naciones Unidas para el Desarrollo. Santiago.

Portales, F. (2000). Chile, Una democracia tutelada. Editorial Sudamericana. Santiago.

Rojas, J. (8 de octubre de 2017). Entrevistado por M. Aranda. Audio digital. Curicó.

Rubilar. L. (7 de octubre de 2017). Entrevistada por M. Aranda. Audio digital. Curicó.

Solorzano, P. (2019). Una experiencia de asesoría en la instalación de los planes de formación ciudadana (Ley 20.911) en establecimientos de dependencia municipal de la Región Metropolitana de Chile. Foro educacional, 32. 53-66. https://doi. org/10.29344/07180772.32.1906

UNESCO (2018). Estado, mercado y currículum escolar: la experiencia chilena (1964-2018). Reflexiones en curso No20 sobre Cuestiones fundamentales y actuales del currículo, el aprendizaje y la evaluación.

Zovato, D. (2014). Las instituciones de la democracia directa. En A. Lissidini, Y. Welp \& D. Zovatto (Compil.) Democracias en Movimiento. Mecanismos de democracia directa y participativa en América Latina (pp. 13-70). Ciudad de México: UNAM. 